\title{
SMART FLES: A METHOD OF MANAGING NON-DETERMINISTIC DATA FOR MULTI-TASKING AND DISTRIBUTED SYSTEMS
}

\author{
Paul D. Keltz, Catherine N. P feil, \\ Melanie H. Okawachi, Edward W. Gertz \\ Cardiac Catheterization Laboratory (111C1) \\ Veterans Administration Medical Center \\ 4150 Clement St., San Francisco, CA. 94121
}

\section{Abstract}

The development of clinical data base structures frequently presents a situation in which the number and type of data items are highly variable or entire data structures require modification over time. Traditional approaches to handling such non-deterministic data (e.g., leaving space for expansion, redefining items no longer used, etc.) have frequently proven to be restrictive and occasionally counter-productive.

Modern multi-tasking computer systems provide an environment in which alternative and flexible data base methodologies are possible. "Smart files" have been developed in our institution to provide a means by which many loosely related computer-assisted functions may access distributed data structures while maintaining independence of the physical implementation of those structures. Secondary benefits of this approach include the ability to distribute data across multiple computer systems and the ability to control access to individual data items being used by multiple functions.

\section{Introduction}

The rate of technological change in modern Medicine is rapid, and perhaps increasing. In some measure this has been due to the increasing application of computer technology to the collection and processing of clinical data and to the availability of computers as decisionsupport tools for physicians. As computers proliferate, they make available vast quantities of raw and processed data. Similarly, computers are used to organize, file and retrieve that data. Thus, it is of importance that the computer and its data base(s) be organized in such a way that:

- useful, or potentially useful data is retained,

- data is stored in a meaningful form,

- data can be retrieved in various combinatorial ways,

- data is protected from destruction and unauthorized access.

There are other considerations, technical and operational, in the construction of computer data bases. The above provide only a framework for consideration of the special type of data base which is the focus of this paper: a data base maintenance system for support of long-term followup of implanted cardiac pacemakers.
Background

Implanted cardiac pacemakers have recently evolved from fixed-capability devices to programmable versions. Older pacemakers were adequately described by a small number of well-understood parameters which were essentially consistent across all manufacturers and models. Current and future programmable pacers vary widely in number of parameters, permissible values and interaction between parameters ${ }^{1,2}$. This is due both to advances in technology and to competition between vendors for new features.

Our existing computer system for pacemaker followup was created when pacemaker parameters were predictable. Due to the advances mentioned above, and also due to major expansion in the scope of the clinic we have had to completely redesign and rebuild the system to cope with nondetermistic data, much larger quantities of data, more users and expanded capabilities for analysis.

There exist numerous published materials describing data base management systems of many different varieties $3,4,5,6$. Computer vendors provide Data Base Management Systems (DBMS) for their equipment ${ }^{7}$, and third-party software vendors do a brisk trade in specialized data base applications programs 6,8. Most commercially available packages are best suited to highly-structured data such as found in commercial applications. Some data base methods and systems do adapt to nondeterministic data, but tend to be available in the form of languages (LISP) or operating systems (MUMPS, UNIX) which were incompatible with our existing hardware and sof tware environment and our need to continue to support existing applications.

At the risk of once again re-inventing the data base wheel, we elected to develop a method of managing nondeterministic data which would be compatible with the operating sustem (RSX11-M V4.0) and high-level language (FORTRAN) already in place.

\section{Environment}

Experience in performing computer-assisted pacemaker followup using highly-structured data elements has given us considerable information about the ways in which the patient records are likely to 
expand ${ }^{9,10}$. Demographic data such as name, address, hospital affiliation, ete. can be handled in a straightforward way. Other data items have a timedependent nature. A patient may have multiple pacemakers implanted over: a period of years. He or she may have the pacemaker lead (the wire connecting to the heart) changed at different times. Each pacemaker is associated with a large number of followup transactions which monitor its function or (in programmable models) change its parameters of operation. In greatly oversimplified form, Figure 1 shows the relationships implicit in these data items.

Experience has also shown the necessity of being able to correlate data across the entire patient population for any given pacemaker model. This provides the opportunity to statistically relate operation of a given pacer model to its predicted norms, and thus identify performance abnormalities which might not be apparent from the record of a single unit. In addition it may be necessary to identify patients by hospital (since many are represented), type of device (in event of FDA action), or other relational elements.

The complexity of the entire data structure of the pacemaker followup system is evident from Figure 1. The figure illustrates entire blocks of data items which correspond to functional entities such as description of a pacemaker, the results of a clinic visit, implantation of a new lead, etc.
Experience dictates that a large amount of sof tware is necessary to properly manipulate data structures of this complexity. The traditional method of accomplishing interface to the data from applications programs would be to provide a library of subprograms which could be called from higher-level language routines ${ }^{7}$. This tends to increase the size of the resulting program by inclusion of many subroutines. We faced another problem in that multiple users of the data base would require simultaneous or nearly simultaneous access. Our multitasking operating system provides the capability for running many copies of programs, but having many programs altering the data can provide an opportunity for corruption ${ }^{4}$.

The RSX operating system also provides for intertask communication, and this feature provided us with a means to resolve the above-mentioned problems. We developed a special "Smart File" program which manipulates the data structures for the applications programs in a controlled and queued manner.

\section{The "Smart File" Program}

The Smart File program is a data structure access method which is highly generalized and controlled by tabular information obtained at the time the program is initiated. In fact there is only one master copy of the smart file program. For each activation a unique name is

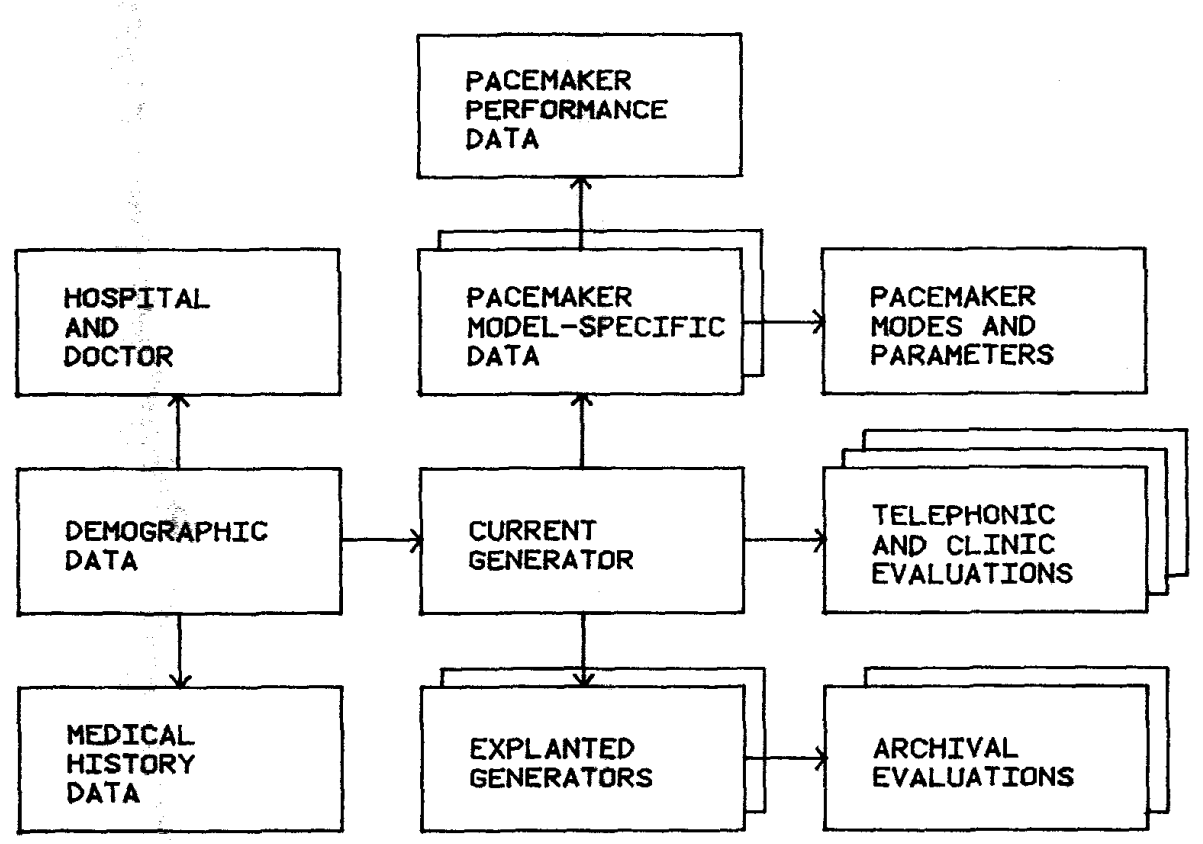

FIGURE 1 
assigned to that task. The running copy of the task obtains its name from the operating system and uses a form of the name to access two physical data files. The first file contains the table of descriptions of the data (a map), the other contains the data itself. Thus multiple Smart Files can coexist with each providing access to different data.

The smart file interfaces to applications programs via the inter-task communication facility of $\mathrm{RSX}-11$. This makes it unnecessary for applications programs to do any data file operations directly, and thereby reduces the task image size. As an added benefit, since inter-task messages are queued by the system on a first-in/first-out basis, access to the data is effectively queued in the same way. Each inter-task message constitutes a complete transaction which obviates problems of concurrency in updating data, pointers, lists, etc. Thus there is rarely a need for traditional locking, exclusion or precedence mechanisms.

An extension of the inter-task message facility permits networking of messages between multiple computer systems, providing for distributed data base operations in a manner transparent to the applications programs (except for a slight increase in the time required for processing the transaction). Tasks which are performing read-type operations are effectively blocked from operation until the $\mathbf{S}$ mart File responds, but tasks performing write-type operations may proceed, thus effecting a high degree of parallelism in data base updating and interactive application activities.

\section{Mapping for Nondeterministic Data}

The concept of using a "map" to describe the form, location and content of data is central to the ability to deal with ongoing changes in the data type and variety. The map for each smart file is loaded at run time from a conventional sequential file. Each record of the map describes the characteristics of one data item in the actual smart file (which is random-access). The elements of these map records are shown schematically in Figure 2. A smart file data record may contain up to 100 separate fields of variable length. Each map record fully describes one of these fields. Thus, by adding map records it is possible to effectively add or redefine fields as needed without modification to the actual computer programs.

As can be seen in Figure 2, each field in the smart file data record has a "name". Applications programs communicating with the smart file customarily refer to data by this name. They may also request from the smart file the map description of the named data item. Thus applications programs may determine the characteristics of data elements on-the-fly. The combination of these capabilities makes it possible for an applications program to operate on data items without prior knowledge of their structure.

For example, in a smart file called CURGEN (which describes the characteristics of a particular patient's pacemaker) there might be a data field called MODEL SPECIFIC PARAMETER. An applications

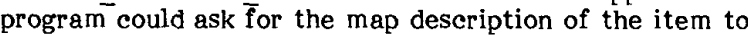
determine the characteristics of this field. It would find (using the example of Figure 2) that this field contains a pointer to an entire record in another smart file. The smart file pointed to (PACERS would contain the description of each different type of pacemaker) would provide the information needed to describe this parameter for this pacemaker. For another patient, with a different pacemaker model, this field would point to a

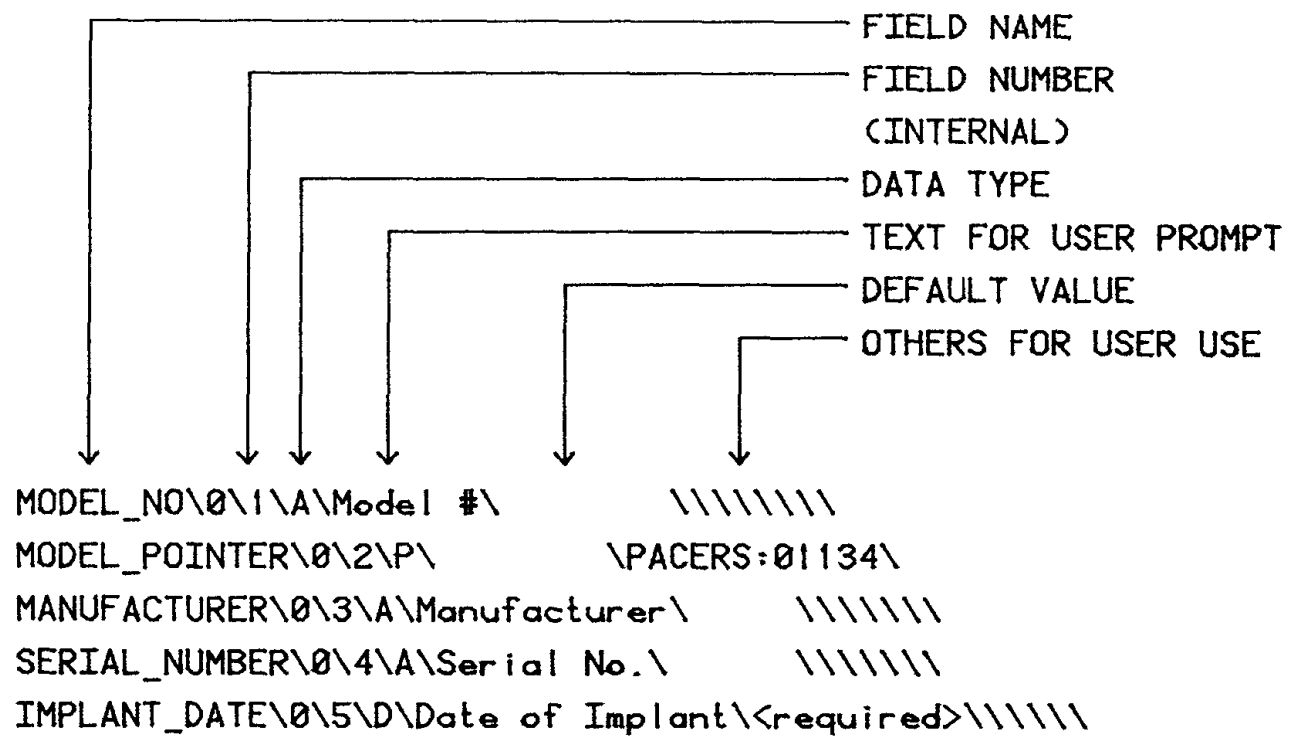

FIGURE 2 
different location in PACERS appropriate to the device. For a patient whose pacemaker had no model specific parameter, the pointer field in CURGEN would be empty so the applications program could avoid asking for unnecessary information. In this way applications programs can be written to use smart file data and maps to control their operation in a user-friendly way.

\section{Threaded Lists}

A frequent need arises in medical data systems to provide data in the form of lists. Examples might be patients referred by a particular hospital, petients having a particular type of pacemaker or all pacemakers having a particular type of battery power source. The smart files provide a means of creating, maintaining and using lists. Fields within each record can be established to contain a type of pointer called a "thread". A thread points to the next logical element in the list, and to the previous element if there is one. Lists are updated by locating the correct position in the list and inserting or deleting an item ${ }^{11,5}$. Mechanisms are included in the smart file functions for 1) adding an item to either end of a thread, 2) adding an item in ascending order, 3) adding an item in descending order, 4) obtaining the location of the next item and 5) deleting an item. These mechanisms support alphabetized lists, calendar date lists and firstcome-first-served lists.

\section{Ongoing Development}

The smart file system for pacemaker followup is still undergoing development. The smart file program is well established as are several utility programs for purposes of maintaining and editing the data structures. Applications programs are still being created for some functions, but progress has been encouraging and we intend to complete full implementation as originally planned. The final system will include more than 10 smart file data structures linked together with pointers and threads as well as a larger number of applications programs which will be serviced by the smart files. Some of the smart files will actually be located in a second computer system and will be accessed via networking to provide journaling of critical transactions as protection from catastrophic hardware malfunction.

\section{Conclusions}

The rapid changes in modern pacemaker technology and the expansion of our pacemaker followup clinic presented a major challenge in the management of large quantities of nondeterministic patient data. We have developed smart files as a method of dealing with changing data without recourse to reprogramming of existing applications software. Our experience with this method during the development process indicates that it fulfills our needs and expectations in a flexible and robust manner.

\section{References}

1. Barold, S.S., Ling, S.O., Falkoff, M.D., Heinle, R.A.: "Programmable Pacemakers: Clinical Indications, Complications and Future Directions" in The Third Decade of Cardiac Pacing, ed: S.S. Barold and J. Mugica, Futura Publishing Company, Mount Kisco, New York, 1982.
2. Calfee, R.V. and Baker, R.G.,Jr: "Multiprogrammable Pacemaker Technology: A Tutorial Review and Prediction" in The Third Decade of Cardiac Pacing, ed: S.S. Barold and J. Mugica, Futura Publishing Company, Mount Kisco, New York, 1982.

3. Fry, J.P. and Sibley, E.H.: "Evolution of Data-Base Management Systems" ACM Computing Surveys $8: 1,1976, \mathrm{p} 7-42$.

4. Tsichritzis, D.C. and Lochovsky, F.H.: Data Base Management Systems Academic Press, New York, New York, 1977

5. Martin, J.: Computer Data-Base Organization Prentice Hall, Inc., Englewood Cliffs, New Jersey, 1975.

6. King, C.H., Strong, R.M. and Goldstein, L.: "Comparing Data Management Systems in Clinical Research: A Survey" Proceedings of the Fourth Annual Symposium on Computer Applications in Medical Care, IEEE, Washington, D.C., 1980, p 1271-1280.

7. PDP-11 Record Management System, RMS-11 User's Guide, Digital Equipment Corp., Maynard, MA, 1979.

8. CLINFO Reference Manual, Bolt, Beranak and Newman, Inc., BBN Research Systems, Cambridge, MA, 1982.

9. Szurek, J.L., Henley, R.R., Gertz, E.W.: "Computer Support System for Pacemaker Surveillance" Presented at the 9th Annual Conference of the Society for Computer Medicine, A tlanta, GA, 1979.

10. Gertz, E.W.: "Types of Surveillance and Who Should Do It" Presented at Current Concepts in Cardiac Pacemakers, Sponsored by the VACO Medical Service and the Southeastern RMEC, Birmingham, AL, Sept. 1980.

11. Knuth, D.E.: The Art of Computer Programming Vol 1. Fundamental Algorithms, Addison Wesley Publishing Co., Reading, MA, 1973. 